\title{
Evaluation of a modified culture medium for Borrelia burgdorferi sensu lato
}

\section{Islay Rodríguez/ ${ }^{+}$, Reto Lienhard*, Lise Gern**, Marie Colette Veuve*, Fatima Jouda**, Hans H Siegrist*, Carmen Fernández, José Enrique Rodríguez}

\author{
National Laboratory of Spirochetes, Tropical Medicine Institute 'Pedro Kourí', PO Box 601, Marianao 13, Havana, Cuba \\ *Laboratory of Microbiology, ADMed, La Chaux-de-Fonds, Switzerland **Laboratory of Eco-Epidemiology of Parasites, \\ Institute of Biology, University of Neuchâtel, Switzerland
}

The aim of the present study was to assess the possible use of a modified medium, prepared in the laboratory using the constituents of Barbour-Stonner-Kelly (BSK) medium and medium 199 as base, for the culture of Borrelia strains, comparing the growth of individual strains in this medium and in the BSK-H medium, and the protein profile and antigenic characteristics of Borrelia proteins expressed in these media. A qualitative evaluation of growth of Borrelia species was made with acceptable results (morphology and motility), but during a quantitative evaluation using the three main genospecies of Borrelia, the better results were obtained with $a$ B. burgdorferi sensu stricto strain. The modified medium did not enable the growth of a $\mathrm{B}$. afzelii strain. The protein profile and antigenic characteristic of the expressed proteins in the modified medium were studied with satisfactory results. These results suggest the modified medium as an alternative for the cultivation of Borrelia strains, with some limitations, in poorly-resourced laboratories.

Key words: Borrelia - new medium - BSK-H medium - proteins

Lyme borreliosis represents the most common vector-borne zoonotic disease in the Northern hemisphere. It is caused by several closely related Borrelia species that are collectively referred to as Borrelia burgdorferi s.l. The human infection is caused primarily by three pathogenic genospecies, B. burgdorferi s.s., B. garinii and $B$. afzelii (Steere 2006). In North America, $B$. burgdorferi s.s. is the sole cause of the infection, while in Europe the three genospecies can cause the disease, and in Asia the latter two species. In addition, nine other closely related species have been identified, but only $B$. lusitaniae, $B$. valaisiana and $B$. spielmani cause human infection. These organisms live in nature in enzootic cycles involving different species of ticks, mainly Ixodes complex (Jouda et al. 2004, Derdákova \& Lencáková 2005, Grubhoffer et al. 2005, Evison et al. 2006, Steere 2006).

Borrelia species can be isolated from different clinical samples, such as skin, blood, and cerebrospinal fluid of patients with early as well as chronic infection indicating their ability to infect different organ and tissue types and to maintain the infection. Isolation of Borrelia species from clinical material is a golden standard for confirming borrelial infection, although it is quite a demanding and long-lasting procedure with limited sensibility (Ruzic-Sabljic \& Strle 2004, Ruzic-Sabljic et al. 2006). Different culture media have been evaluated

${ }^{+}$Corresponding author: islay@ipk.sld.cu

Received 28 May 2007

Accepted 23 November 2007 and used for cultivation, but most frequently BarbourStonner-Kelly (BSK) medium (Gorelova \& Shcherbakov 1991, Marques et al. 2000, Ruzic-Sabljic \& Strle 2004).

The aim of the present work was to assess the possible use of a modified medium for the culture of Borrelia strains comparing the growth of individual strains of B. burgdorferi s.s., B. garinii and B. afzelii in this medium and in medium BSK-H prepared according to Sinsky and Piesman (1989). We also analyzed the protein profile and antigenic characteristics of Borrelia proteins expressed in the modified medium.

The modified medium was prepared using the constituents of BSK medium and medium 199 as base in substitution of medium CMRL 1066, following the original recipe (Barbour 1984). Briefly, $5 \mathrm{~g}$ of neopeptone (Difco Laboratories, USA), $50 \mathrm{~g}$ of bovine serum albumin (BSA) (fraction V) (Sigma Chemical Co, USA), $6 \mathrm{~g}$ of N-2-hydroxyethylpiperazine-N'-2-ethanesulphonic acid (BDH Laboratory Supplies Poole, England), $0.7 \mathrm{~g}$ of bi-hydrated tri-sodium citrate $(\mathrm{BDH}), 5 \mathrm{~g}$ of $\mathrm{D}(+)$ anhydrous glucose (BDH), $0.8 \mathrm{~g}$ of sodium pyruvate (BDH), $0.4 \mathrm{~g}$ of N-acetyl-D-glucosamine (Sigma), $2.2 \mathrm{~g}$ of sodium bicarbonate (Sigma), and $2.53 \mathrm{~g}$ of TC yeastolate (Difco) were added to $900 \mathrm{ml}$ of milli-Q water. The solution was slowly stirred until total suspension of the ingredients, then $100 \mathrm{ml}$ of medium 199 (10X) with L-glutamine (GibcoBRL, USA) was added. The medium was adjusted to $\mathrm{pH} 7.6$ with $1 \mathrm{~N} \mathrm{NaOH}$, and $64 \mathrm{ml}$ of heat-inactivated rabbit serum (in house-preparation) was added, then it was sterilized by filtration through a 1.2-0.45-0.22 $\mu \mathrm{m}$ filters (Millipore Corp). Finally, $200 \mathrm{ml}$ of gelatin 7\% (Oxoid, England) sterilized by autoclave was added. After preparation, $6.0 \mathrm{ml}$ aliquots of medium were dispensed into polystyrene culture tubes (13 by $100 \mathrm{~mm}$ ) (PolyLabo, France). 
BSK-H medium was prepared in parallel using BSK$\mathrm{H}$ medium (Sigma), heat-inactivated rabbit serum (Sigma) and gelatin (Difco). Amino acids and antibiotics were added according to Sinsky and Piesman (1989). The medium was aliquoted as mentioned above.

A qualitative evaluation of Borrelia growth was carried out using B. garinii (NE83) and B. valaisiana (NE4555) obtained from free-living I. ricinus ticks collected in Switzerland. The number of spirochetes per milliliter was determined with a counting chamber (Helber's cells). Identical volumes with the same concentration of each strain were added to duplicate tubes containing the media and incubated for 10 days at $35^{\circ} \mathrm{C}$, and motile and uniformly refractile spirochetes were observed in both media by dark-field microscopy, but the cellular concentration was lightly superior in BSK$\mathrm{H}$ medium. Later, a quantitative evaluation for comparing Borrelia growth was done. Three low-passages (2 passages) B. burgdorferi s.l. strains were randomly selected from the collection of the isolates of the laboratory of Eco-Epidemiology of Parasites at the Institute of Biology of the University of Neuchâtel (Switzerland). B. burgdorferi s.s. (NE4502), B. garinii (NE4546) and $B$. afzelii (NE4505) were obtained from free-living $I$. ricinus ticks collected in Switzerland. Strains were identified to species level by polymerase chain reaction/restriction fragment length polymorphism analysis as previously described (Postic et al. 1994) or by reverse line blot (Poupon et al. 2006). Inoculum volumes of each strain were determined, using the counting chamber, for a final concentration in the fresh media of $1.5 \times 10^{4}$ spirochetes $/ \mathrm{ml}$. The media were inoculated simultaneously, 10 replicas of each strain were made for the modified medium and five replicas for the BSK-H medium. All the media were incubated to $34^{\circ} \mathrm{C}$. To asses the growth of individual strains in BSK-H and modified medium, the number of cells was determined 2, 4, 7, 10, 14, and 23 days after inoculation using the counting chamber and curves of growth were done (Fig. 1) with the mean values of concentration of each strain. Dark field microscopy was used in order to detect any contamination in the media and to check the morphology and motility of borrelias. No differences in the latter aspects were observed between the media.

The logarithmic phase for B. burgdorferi s.s. in the modified medium was slower than in BSK-H, but the concentrations of spirochetes during the stationary phase in both media were similar, and the number of borrelias in the decline phase was superior in the modified medium.

The results differed for the other Borrelia species. In the modified medium the number of $B$. garinii cells was lower than in BSK-H until day 14, the logarithmic phase was very slow and the stationary phase was not clearly observed, and the decline phase was similar in both. The multiplication of B. afzelii strain in the modified medium was not possible; we only observed some borrelias with reduced motility. The number of cells at the beginning of culture was relatively low, but we used this one because it enables a quantitative approach to the number of spirochetes in the samples.
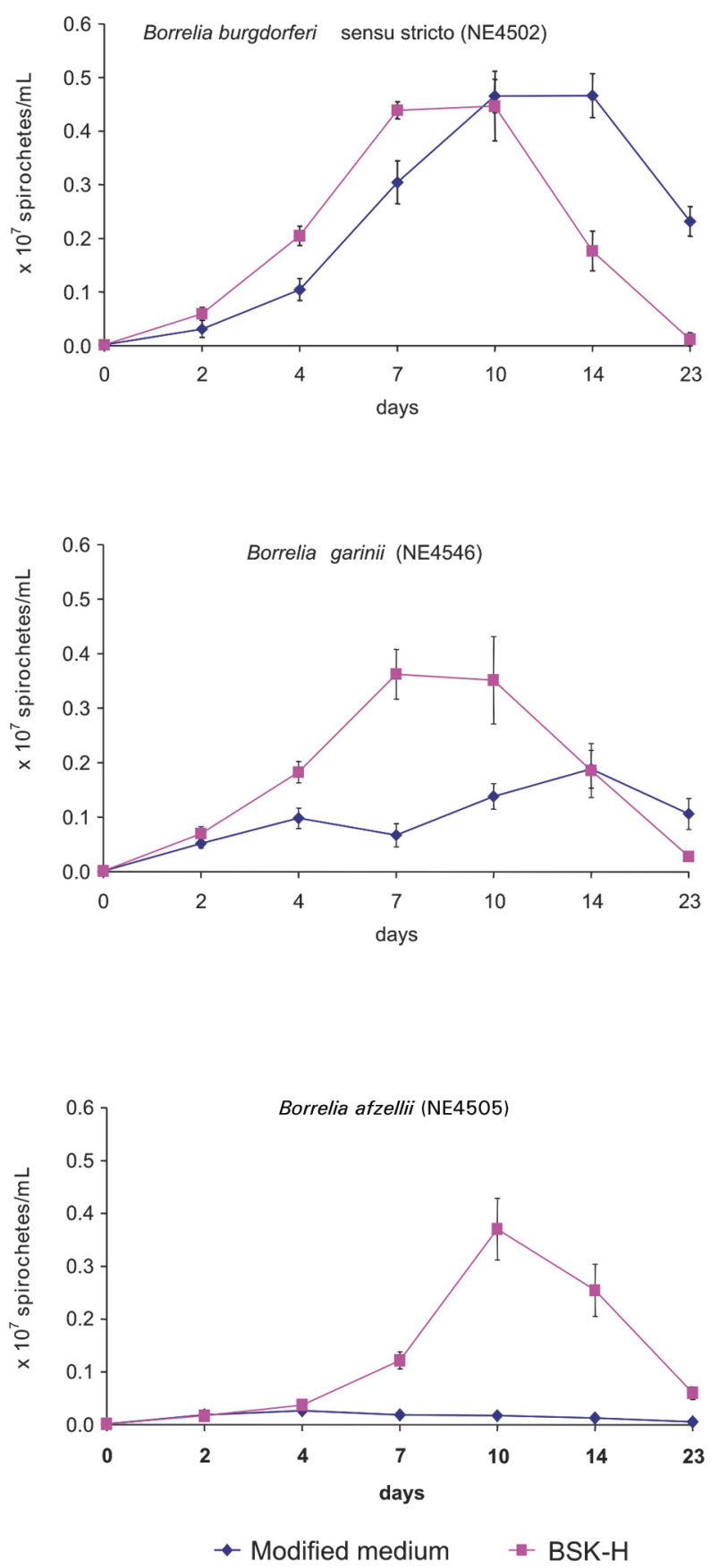

Fig. 1: growth curves of Borrelia strains in different media.

The growth of Borrelia strains differed also in the BSK-H medium and not only in the modified medium prepared with medium 199. Strains from different Borrelia species grew differently, maybe due to the fastidious nature of the organism and changes that Borrelia must undergo to adapt the transition from living biological material to an artificial medium, since the Borrelia strains had received only two passages starting from the primary isolation. 
It is known that borrelias are microorganisms fastidious for growth in defined chemical media, and changes of batches and/or commercial house of medium constituents affect the ability of the medium to growth B. burgdorferi s.l. It has been demonstrated, for example, that the fraction V BSA can affect the recovery of low numbers of borrelias (Callister et al. 1990).

Medium ingredients and other factors including temperature of incubation, $\mathrm{pH}$ of the medium, and capacity of particular borrelial species can also influence on the borrelial growth (Ruzic-Sabljic \& Strle 2004). In this study we only modified the composition of medium. When comparing the CMRL 1066 medium (GibcoBRL, USA) with medium 199 (GibcoBRL, USA), no significant differences were found in relation to inorganic salts, amino acids and vitamins composition; however, the concentration of amino acids in medium 199 was higher and the chemical composition of other non-essential components for Borrelia differed from one medium to the other.

Another purpose of the present work was to analyze the protein profile of a Borrelia strain in the modified medium and to know the antigenic properties of expressed proteins. A culture of $B$. garinii (NE83) in the modified medium during 10 days at $34^{\circ} \mathrm{C}$ was made. The cells were harvested, washed and lysed in Laemeli buffer containing Tris- $\mathrm{HCl} 0.5 \mathrm{M}$ buffer, sodium dodecyl sulfate, 2-mercaptoethanol, glycerol and bromophenol blue, and finally the spirochetes were boiled for $3 \mathrm{~min}$. Electrophoresis was performed in polyacrylamide gels $(12.8 \%$ acrylamide running gel and $6 \%$ acrylamide stacking gel) as described by Laemeli (1970). The gels were transferred to nitrocellulose membranes, one membrane was stained with colloidal gold and the other was used for a Western blot-IgG (home-made) with a human positive control serum to Lyme Borreliosis by ELISA (IgM/IgG) (BioMérieux), and Western blot-IgG with B. garinii, B. burgdorferi s.s. and B. afzelii antigens. The results are shown in Fig. 2.

The major Borrelia proteins (specific and non-specific) were expressed by $B$. garinii in this modified medium according to colloidal gold stain picture. The results of Western-blot analysis of whole-cell lysates of $B$. garinii probed with the human immune serum demonstrated that a large number of expressed proteins are antigenic, which did not change when the Borrelia grew in different media. However, some differences in the region of 20-25 $\mathrm{kDa}$ proteins (presumably OspC) were observed. Considerable antigenic variations have been documented for Borrelia strains; besides the adaptive mechanisms of the spirochetes that influence protein expression, each strain has its own potential to express a particular protein (Wilske et al. 1996, Ruzic-Sabljic et al. 2001). According to these results, the antigen obtained could be used in a Western-blot for the diagnosis of Lyme borreliosis in humans.

To be useful for laboratory purposes, the medium must be stable, available and efficacious in supporting the growth of all human pathogenic Borrelia strains (Ruzic-Sabljic \& Strle 2004), including B. lusitaniae, $B$. valaisiana and B. spielmani; this latter element was

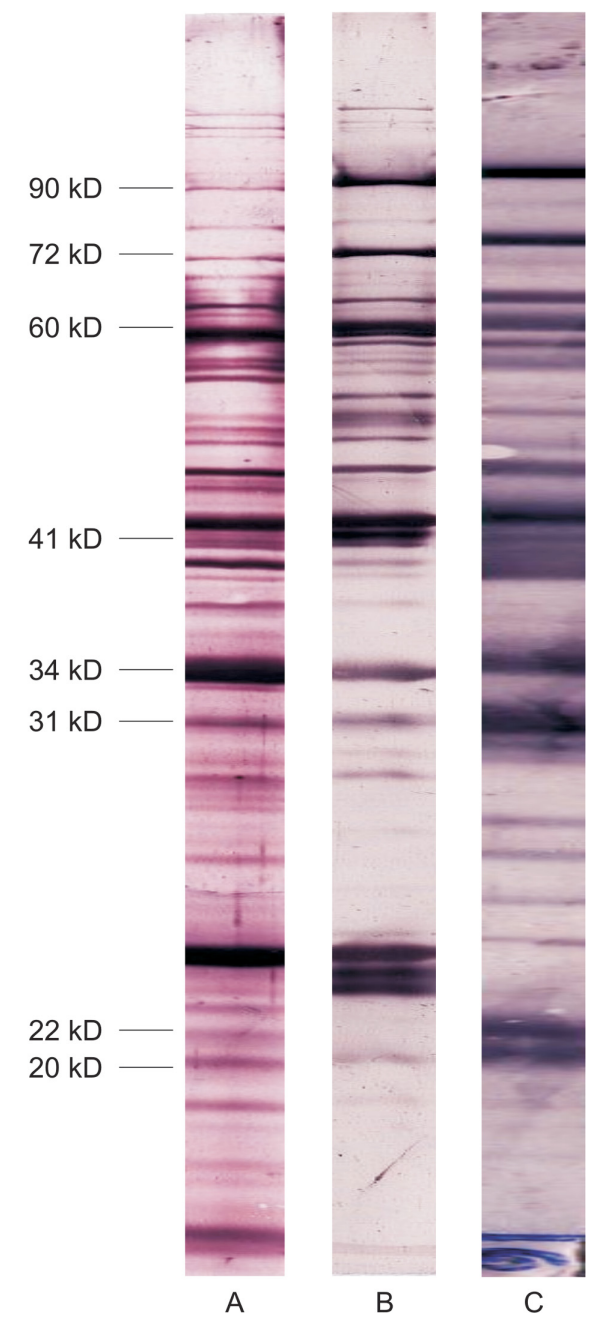

Fig. 2: whole-cell lysates of Borrelia garinii (NE 83) from different media. A: lysate from the modified medium with colloidal gold stain. B: western blot of lysate from the modified medium. C: western blot of lysate from BSK-H medium.

not well defined in this work, because the main limitation of the present study was the low number of used strains. Further works with a larger number of strains is needed to compare the results of this study on the differences in the growth of Borrelia species in the modified and BSK-H medium (Sinsky \& Piesman 1989).

The modified medium evaluated in the present study could be an alternative for the culture of B. burgdorferi s.l. strains taking into account its limitation for some strains in Latin-American and Caribbean countries with poorlyresourced laboratories, although there are not much published papers about the presence of Borrelia genospecies in this region, only serological evidences of Lyme disease have been published (Ciceroni et al. 1994, Abel et al. 2000, Rodríguez et al. 2004, G Gordillo et al. unpublished observatious). 


\section{ACKNOWLEDGEMENTS}

To Swiss Confederation for providing the financial support of the fellowship. To the staff of the laboratory of Eco-Epidemiology of Parasites (University of Neuchâtel, Switzerland) and to the staff of Laboratory of Microbiology, ADMed (La Chaux-deFonds, Switzerland) whose collaborations were very important to the execution of the present study.

\section{REFERENCES}

Abel IS, Marzagao G, Yoshinari NH, Schumaker TT 2000. Borrelia-like spirochetes recovered from ticks and small mammals collected in the Atlantic Forest Reserve, Cotia county, State of São Paulo, Brazil. Mem Inst Oswaldo Cruz 95: 621-624.

Barbour AG 1984. Isolation and cultivation of Lyme disease spirochetes. Yale J Biol Med 57: 521-525.

Callister SM, Case KL, Agger WA, Schell RF, Johnson RC, Ellingson JLE 1990. Effects of bovine serum albumin on the ability of Barbour-Kelly medium to detect Borrelia burgdorferi. J Clin Microbiol 28: 363-365.

Ciceroni L, Bartoloni A, Guglielmetti P, Paradisi F, Barahona HG, Roselli M, Ciarrocchi S, Cacciapuoti B 1994. Prevalence of antibodies to Borrelia burgdorferi, Borrelia parkeri and Borrelia turicatae in human settlements of the Cordillera Province, Bolivia. J Trop Med Hyg 97: 13-17.

Derdákova M, Lencáková D 2005. Association of genetic variability within the Borrelia burgdorferi sensu lato with the ecology, epidemiology of Lyme borreliosis in Europe. Ann Agric Environ Med 12: 165-172.

Evison J, Aebi C, Francioli P, Péter O, Basseti S, Gervaix A Zimmerli S, Weber R 2006. Borréliose de Lyme 1ére partie: épidémiologie et diagnostic. Rev Med Suisse 2: 919-924.

Gorelova NB, Shcherbakov SV 1991. Medium modification for the cultivation and isolation of the agent of Lyme's disease. Med Parazitol 3: 56-57.

Grubhoffer L, Golovchenco M, Vancová M, ZacharovováSlavícková K, Rudenko N, Oliver JH 2005. Lyme Borreliosis: insights into tick-/host-Borrelia relations. Fol Parasitol 52: 279-294.

Jouda F, Perret JL, Gern L 2004. Density of questing Ixodes ricinus nymphs and adults infected by Borrelia burgdorferi sensu lato in Switzerland: spatio-temporal pattern at a regional scale. Vect Borne Zoon Dis 4: 23-32.

Laemeli UK 1970. Cleavage of structural proteins during the assembly of the head of bacteriophage T4. Nature 227: 680-685.

Marques AR, Stock F, Gill V 2000. Evaluation of a new culture medium for Borrelia burgdorferi. J Clin Microbiol 38: 4239-4241.

Postic D, Assous MV, Grimont PA, Baranton G 1994. Diversity of Borrelia burgdorferi sensu lato evidenced by restriction length polymorphism of $\operatorname{rrf}(5 \mathrm{~S})$-rrl (23S) intergenic spacer amplicons. Int J Syst Bacteriol 44: 743-752.

Poupon MA, Lommano E, Humair PF, Douet V, Rais O, Schaad M, Jenni L, Gern L 2006. Prevalence of Borrelia burgdorferi sensu lato in ticks collected from migratory birds in Switzerland. Appl Environ Microbiol 72: 976-979.

Rodríguez I, Fernández C, Cinco M, Pedroso R, Fuentes O 2004. Do antiborrelial antibodies suggest Lyme disease in Cuba? Emerg Infect Dis 10: 1698-1700.

Ruzic-Sabljic E, Arnez M, Lotric-Furlan S, Maraspin V, Cimperman J, Strle F 2001. Genotypic and phenotypic characterization of Borrelia burgdorferi sensu lato strains isolated from human blood. J Med Microbiol 50: 896-901.

Ruzic-Sabljic E, Lotric-Furlan S, Maraspin V, Cimperman J, Logar M, Jurca T, Strle F 2006. Comparison of isolation rate of Borrelia burgdorferi sensu lato in MKP and BSK-II medium. Int J Med Microbiol 296: 267-273.

Ruzic-Sabljic E, Strle F 2004. Comparison of growth of Borrelia afzelii, B. garinii, and B. burgdorferi sensu stricto in MKP and BSK-II medium. Int J Med Microbiol 294: 407-412.

Sinsky RJ, Piesman J 1989. Ear punch biopsy method for detection of Borrelia burgdorferi from rodents. J Clin Microbiol 27: 1723-1727.

Steere AC 2006. Lyme borreliosis in 2005, 30 years after initial observations in Lyme Connecticut. Wien Klin Wochenschr 118: 625-633.

Wilske B, Busch U, Eiffert H, Fingerle V, Pfister HW, Rossler D, Preac-Mursic V 1996. Diversity of OspA and OspC among cerebrospinal fluid isolates of Borrelia burgdorferi sensu lato from patients with neuroborreliosis in Germany. Med Microbiol Immunol 184: 195-201. 